\title{
Prevalence of age related macular degeneration in persons aged 50 years and over resident in Australia
}

Reg A Mitchell

\begin{abstract}
Study objective-The aim was to determine, employing non-invasive procedures, the prevalence of age related macular degeneration in persons 50 years of age and over.

Design-A clinical investigation and a retrospective examination of ophthalmological records were employed in this study.

Setting-The study was conducted over the period 1988 to 1990 in the Western Metropolitan Health Region of New South Wales, Australia.

Participants-A total sample of 3283 subjects stratified by local government area, age, and sex was obtained from the source population. It proved possible to confirm ophthalmological diagnoses in only 2522 of these subjects.

Main results-All data were collected using accepted ophthalmological procedures and all diagnoses were confirmed through the use of independently derived ophthalmological records. A total of 428 subjects $(13 \cdot 0 \%)$ had a confirmed symmetrical diagnosis of age related macular degeneration. A total prevalence for diseased eyes of $14.9 \%$ was obtained. Prevalence of diseased eyes rose from $10.4 \%$ in those 50 to 64 years of age to $31.0 \%$ in those 85 years of age and over.

Conclusions-There are several sources of error which can affect such a large sample study and are identified. Despite these, the prevalence rates obtained in this study provide normative rates for age related macular degeneration for persons 50 years of age and over.
\end{abstract}

f Epidemiol Community Health 1993; 47: 42-45

Studies of age related macular degeneration have reported quite varied prevalence rates for this visually disabling disease. Several studies have reported rates computed from data derived from various samplings of the Framingham heart study cohort. ${ }^{1-4}$ Variability in the prevalence rates derived from this cohort can be attributed to differing definitions of the disease, restricting the sample age range (52 to 85 years of age), and requiring subjects to present with a Snellen acuity of $6 / 9$ or less before undergoing a fundus examination.

Other studies have computed rates for age related macular degeneration from samples which did not have an upper age limit. ${ }^{5}$ However, in both of these studies the samples were specialised, self selecting, and either had a pre-existing medical condition or were attending an ophthalmological clinic.

Irrespective of the source of the sample and the associated errors of measurement, definition of disease, and restricted age range, all research on age related macular degeneration shows an increase in prevalence with age. The prevalence findings across age groups for the studies cited above are summarised in table I.

Each of the above studies presents with one or more limitations which prevent generalisations of their findings to the elderly population 50 years of age and over. These limitations can be categorised into three generic areas; the manner in which the disease is defined, the inclusion of a certain Snellen visual acuity criterion as a sign of the disease, and finally the nature of the sample used in the specific study. These three general limitations lead to some question as to the accuracy of the prevalence rates presented for age related macular degeneration. The aim of the present study was to determine, employing non-invasive procedures, the prevalence of this disorder in persons 50 years of age and over.

\section{Methods}

The base population was comprised of persons 50 years of age and over. From this base population the source population of residents in the Western Metropolitan Health Region of New South Wales was selected. The source population was stratified into 14 local government areas and then into age groups of 50 to 64 years, 65 to 74 years, 75 to 85 years, and over 85 years. From this population a $2.5 \%$ sample for the three age strata over 64 years of age was selected. Had this same percentage been applied to those in the age stratum 50 to 64 years a sample for this group alone of 3942 persons would have to have been obtained, which would have required a prohibitive amount of time to assess. Consequently a $1.0 \%$ sample was drawn from this stratum. This reduction in sample size yielded an increase in the standard error from 0.69 to 0.79 . Thus the projected sample used in this study consisted of a total of 3251 persons $(1.37 \%)$, 1460 males and 1791 females, from the source population.

A list of registered general medical practitioners for each local government area was generated. Grids of area $50 \mathrm{~m}^{2}$ were imposed on maps of each local government area. These grids only covered indicated residential regions of the areas concerned. The grids on each map were then numbered sequentially, starting with the upper left hand corner and proceeding down the map, ie, 
Table I Age specific percentage prevalence rates for age related macular degeneration

\begin{tabular}{|c|c|c|c|c|c|c|}
\hline \multirow[b]{2}{*}{ Authors } & \multicolumn{4}{|c|}{ Age strata (years) } & \multirow[b]{2}{*}{ Total } & \multirow{2}{*}{$\begin{array}{l}\text { Limitation } \\
\text { (See key below) }\end{array}$} \\
\hline & $50-64$ & $65-74$ & $75-85$ & $85+$ & & \\
\hline \multirow{3}{*}{$\begin{array}{l}\text { Kahn et al (1977) } \\
\text { Leibowitz et al } \\
(1980)^{2} \\
\text { Sperduto and Seigel } \\
(1980)^{3} \\
\text { Podgor et al }(1983)^{4}\end{array}$} & 1.6 & $11 \cdot 0$ & $12 \cdot 1$ & * & $8 \cdot 8$ & $1,2,3$ \\
\hline & $1 \cdot 2$ & $6 \cdot 4$ & $19 \cdot 7$ & $\star$ & $5 \cdot 7$ & $1,2,3$ \\
\hline & $35 \cdot 0$ & $47 \cdot 0$ & $50 \cdot 0$ & * & $41 \cdot 0$ & 1,3 \\
\hline \multirow{3}{*}{$\begin{array}{l}\text { Martinez et al } \\
(1982)^{5} \\
\text { Mitchell and Sarks } \\
(1984)^{6}\end{array}$} & $17 \cdot 6$ & $27 \cdot 4$ & $43 \cdot 0$ & $\star$ & $28 \cdot 8$ & 1,3 \\
\hline & $t$ & $2 \cdot 1$ & $11 \cdot 44$ & $22 \cdot 84$ & $14 \cdot 4$ & $1,2,3$ \\
\hline & $14 \cdot 7 \ddagger$ & $18 \cdot 8$ & $27 \cdot 7$ & $38 \cdot 30$ & $22 \cdot 3$ & 3 \\
\hline
\end{tabular}

*No subjects over 85 years of age included in the study

tNo subjects under 65 years of age included in the study

fincludes subjects 50 to 64 years of age

Key to limitations: 1 Definition of disease

2 Snellen acuity criterion

3 Nature of sample

north to south. Using tables of random numbers, locations of the residences of a possible random sample were then placed on the grid of each local government area.

To ensure achievement of the projected sample sizes an excess of $20 \%$ over the projected sample size was placed on each local government area. Similarly a random sample of practitioners or practices was generated. Each sampled practitioner/practice was then contacted, the author's credentials and affiliations presented, and the nature of the study described. Practices and/or practitioners were requested to assist with this study by permitting the author access to all patients 50 years of age and over who attended that practice.

Practitioners were requested to contact potential subjects, explain the nature of the study to them, and seek their consent for the author to contact them directly. Subjects agreeing to participate and falling within the predetermined locations were included in the study. Those agreeing to participate but falling outside the predetermined locations were contacted by the author and thanked for agreeing to participate but were excluded from the study.

Data collected using the study protocol included biographical data such as name, sex of the subject, identification number (supplied by the author), age in years at the date of examination, occupation (either present or immediately prior to retirement), current address, postcode, and local government area. These latter three variables were used primarily to ensure integrity of the sample and that the proportions projected in the sampling frame would be adhered to.

Next a basic history of eye problems was taken: whether or not spectacles were worn and if so the nature of the optical correction. The nature of any such correction was objectively classified as minus lenses (for myopic correction), plus lenses (for hypermetropic correction or presbyopia), cylindrical lenses (for astigmatic correction), or multifocal (bifocal, trifocal) or variable focus lenses.

Subjects were next asked whether or not they had been seeing a professional person in relation to their vision care, and the name and profession of that person. Where subjects had seen an eye care specialist they were asked to sign a consent form giving the author permission to contact that professional and examine that patient's ophthal- mological records. All such records were to be examined. The purpose of establishing contact with the ophthalmologist was to confirm diagnoses claimed by the subject.

Each subject was asked to provide information as to any known current ocular condition. Specific conditions recorded, for each eye, were: trauma, glaucoma, retinal detachment, age related macular degeneration, thyroid eye disease, congenital eye disease, diabetes, and cataract. Where discrepancies existed between subject's stated ocular condition and an ophthalmologist's diagnosis the latter was recorded as the principal diagnosis.

In the context of this study the term "principal diagnosis" was applied as follows. In the presence of multiple visual pathologies the disease which is, in the opinion of the ophthalmologist, the principal cause of reduced visual acuity or a visual disability will be designated as the "principal diagnosis". Further if the signs and/or symptoms of only one diseae are recorded this will be assumed to be the "principal diagnosis".

Data from the protocol were coded for computer analyses and the Statistical Package for the Social Sciences (SPSS) program was used to obtain the majority of the statistical results. Additional analyses were carried out, using SPSS generated summary statistics, on the author's personal computer employing programs written by the author in compiled extended BASIC.

\section{Results}

The total size of the obtained sample was 3283 persons 50 years of age and over (compared with an expected total size of 3251 persons). This obtained sample was comprised of 1478 (projected 1460) males and 1805 (projected 1791) females. This sample yielded a $1.38 \%$ sampling of the source population. From this source population of persons 50 years of age and over resident in the Western Metropolitan Health Region of New South Wales, $1.32 \%$ of the males and $1.43 \%$ of the females were sampled in this study.

In 118 subjects it proved impossible to verify diagnoses from records as they had not seen an ophthalmologist in the previous three years. For the 3165 subjects whose diagnoses were obtained it was found that 2620 diagnoses were made by ophthalmologists and the remaining 545 may have been made by an ophthalmologist, general practitioner, or some other eye care specialist. For the 2620 subjects in whom the diagnosis was made by an ophthalmologist it proved possible to confirm these diagnoses with the specialist for 2522 subjects. Diseases and/or conditions stated were recorded for both eyes. Where any discrepancy existed between the practitioner's records and the subject's responses then, with some exceptions, the practitioner's diagnoses were accepted and recorded. The exceptions to this were for those cases where the subject had recently been seen by an ophthalmologist and the results of this examination had not, as yet, been received by the referring practitioner.

The most conservative estimate of disease prevalence will be obtained by employing only those subjects whose diagnoses were confirmed from ophthalmological records (2522) on the 
numerator with the total sample size (3283) as the denominator.

A total of 428 subjects had a confirmed symmetrical diagnosis and 124 had asymmetrical diagnoses of age related macular degeneration. The prevalence for bilateral cases was thus computed as $13.0 \%$. The total prevalence was found to be $16 \cdot 8 \%$. The prevalence rate for eyes with the condition was $14.9 \%$.

\section{Discussion}

The rates computed above are considered to be point estimates. In order to compensate, in part, for the error inherent in point estimates, $95 \%$ and the $99 \%$ confidence intervals for each of the above point estimates are given below.

For bilateral cases the $95 \%$ confidence interval was computed to be $11 \cdot 8-14 \cdot 2 \%$ and the $99 \%$ confidence interval was $11 \cdot 5-14.5 \%$. The corresponding confidence intervals when persons with the disease were considered were $15 \cdot 5-18 \cdot 1 \%$ (95\% confidence interval) and $15 \cdot 1-18.5 \%(99 \%$ confidence interval). Finally for eyes with the disease the $95 \%$ confidence interval was found to be $14 \cdot 0-15 \cdot 8 \%$ and the $99 \%$ confidence interval $13 \cdot 8-16 \cdot 0 \%$.

When the numbers of cases with age related macular disease as their principal diagnosis were examined across age strata (table II) the relationship of the prevalence of this disease to age became even more apparent.

The prevalence rate for age related macular disease increased from $9.6 \%(10.4 \%$ for eyes) in the youngest age group to $25.7 \%$ (31.0\% for eyes) in the oldest age group. The rates in table II indicate that the percentage of those with healthy eyes declined with increasing age and that there was a concomitant increase in those eyes with macular degeneration. This relationship between the prevalence of macular degeneration and age supports the findings of earlier studies. ${ }^{256}$

\section{SOURCES OF ERROR}

Three principal sources of such error have been identified in this study: those errors associated with the sampling procedure and the sample itself, observer errors, and errors associated with the diagnosis of age related macular degeneration.

\section{Sampling errors}

The ethical considerations which pertain, of necessity, to present day research demand that all human subjects, among other considerations, give informed consent to participate as a subject in a research study. The implication of this consideration is clear; essentially all subjects who give such consent constitute a sample of volunteers. Not all persons in a given target population would so

Table II Frequencies, percentage prevalence and standard errors (in parentheses) for normals and persons with age related macular degeneration within age groups for both total persons and total eyes

\begin{tabular}{|c|c|c|c|c|c|c|c|c|c|c|}
\hline \multirow[b]{2}{*}{$\begin{array}{l}\text { Age } \\
\text { stratum } \\
\text { (years) }\end{array}$} & \multicolumn{5}{|c|}{ Total persons } & \multicolumn{5}{|c|}{ Total eyes } \\
\hline & $\begin{array}{l}\text { Nor } \\
\text { (bild } \\
\text { cases } \\
n\end{array}$ & & $\begin{array}{l}\text { Mc } \\
\text { dis } \\
n\end{array}$ & $\%$ & (SEM) & $\begin{array}{l}\text { Norm } \\
n\end{array}$ & $\begin{array}{l}l s \\
\%\end{array}$ & & $\%$ & (SEM) \\
\hline $\begin{array}{l}50-64 \\
65-74 \\
75-84 \\
85+\end{array}$ & $\begin{array}{r}572 \\
323 \\
59 \\
5\end{array}$ & $\begin{array}{r}41 \cdot 4 \\
27 \cdot 2 \\
12 \cdot 2 \\
4 \cdot 4\end{array}$ & $\begin{array}{r}132 \\
176 \\
91 \\
29\end{array}$ & $\begin{array}{r}9 \cdot 6 \\
14 \cdot 8 \\
18 \cdot 8 \\
25 \cdot 7\end{array}$ & $\begin{array}{l}(0 \cdot 79) \\
(1 \cdot 03) \\
(1 \cdot 78) \\
(4 \cdot 11)\end{array}$ & $\begin{array}{r}1366 \\
649 \\
121 \\
7\end{array}$ & $\begin{array}{r}49 \cdot 5 \\
27 \cdot 3 \\
12.5 \\
3.1\end{array}$ & $\begin{array}{r}287 \\
390 \\
212 \\
70\end{array}$ & $\begin{array}{l}10.4 \\
16.4 \\
21.9 \\
31.0\end{array}$ & $\begin{array}{l}(0.58) \\
(0.76) \\
(1.33) \\
(3.08)\end{array}$ \\
\hline
\end{tabular}

volunteer and yet random sampling requires all subjects in the target population to have an equal chance of inclusion in any sample. Realistically, in describing the target population of this study the term volunteer must be applied.

This element of obtaining "volunteers" is further accentuated by the fact that in the first stage of the sampling procedure, general practitioners, on the author's behalf, sought consent from potential subjects. The author has no way of estimating the refusal rate associated with this process. For ethical reasons, refusal by a practitioner's patients was not to be communicated to the author. A further source of error may have been generated by the practitioners' selection bias, ie, unwittingly selecting "suitable" patients to volunteer as subjects.

This use of general practitioners as the "first point of contact" with potential subjects also contributed to further unknown sources of error in the sample. There is no guarantee that all elderly persons in the target population saw a general practitioner over the period of this study. While proportions of subjects in the target population who had not seen a general practitioner are unknown, the $3.6 \%$ of the sample who had not seen an eye care specialist in the preceding three years is suggestive that some persons in the target population would not have seen a general practitioner.

Another consideration with respect to the sample is that not all persons attend a general practitioner or specialist within their local government area or health region. Of concern here would be the unknown number of suitable subjects excluded from the sample due to their attending a practitioner outside the Western Metropolitan Health region.

As a consequence of the above, non-volunteers, persons who had not seen a general practitioner, and persons who attended a practitioner outside the Western Metropolitan Health Region of New South Wales would automatically be excluded from the target population. An estimate of overall sampling error associated with a stratified sample was obtained employing the procedure described by Yates (1960). ${ }^{7}$ This procedure, appropriate to sampling with probabilities proportional to the size of unit, yielded an overall error of $0.42 \%$.

\section{Observer errors}

It is clear that this study employed differential sampling rates by age. Throughout the discussion of prevalence, unweighted rates were presented and discussed. It was found that, given the large sample size, differences between weighted and unweighted prevalence rates were of the order of $0.01 \%$. When corrected to one decimal place there was no difference between the weighted and unweighted rates.

The procedures and protocols for data collection employed in this study are founded on well established objective clinical procedures. There are, however, those generally accepted errors which can be associated with the use of medical records. All ophthalmological diagnoses were obtained from such records. The researcher had no control over errors associated with recording and is unaware of limitations, biases, or deficiencies in such records. 
The confidence intervals for the various prevalence rates associated with age related macular degeneration presented above give some estimates of the error in the specific point prevalence rates. There are, however, several sources of uncontrolled error which must be considered if these prevalence rates are to be applied.

\section{Errors in diagnosis}

In this present study the number of ophthalmologists consulted constituted $10.5 \%$ of all practitioners in New South Wales. ${ }^{8}$ All ophthalmologists were Fellows of the Royal Australian College of Ophthalmologists (RACO). This College maintains uniform standards of practices and procedures through its prefellowship training programme. RACO also has a vigorous in-service and continuing education programme manifested by its annual conferences, regional conferences, speciality conferences and workshops, publication of an authoritative and highly regarded journal, a regular newsletter, and through the production of audiovisual materials available to fellows, members and associates.

The above uniformity in training and further education does not preclude this research from errors in diagnosis. There is not only the ever present likelihood of interobserver errors but also the likelihood of intraobserver error in making any medical diagnosis. When a disease such as age related macular degeneration is being studied very subtle signs may be either overlooked or considered to be too insignificant (in the presence of other disease) to warrant recording. The histopathological signs of age related macular disease may only be observable at necropsy. The author trusted that ophthalmologists employed standard practices and procedures in diagnosing age related macular degeneration; however the possibility of variability must be recognised as a source of error.

The definition employed in this study for "principal diagnosis" was presented above. When only one pathology is present the principal diag- nosis is not in doubt. In the presence of multiple pathologies it is a matter of professional opinion as to what constitutes the "principal" diagnosis. The author had to rely upon the ophthalmologists' expertise and records in determining this matter. There is thus a source of error associated with any subjectivity in assigning "principal" diagnosis.

It must be noted that adherence to the use of principal diagnoses in this study may well have resulted in an underestimate of the prevalence of age related macular degeneration. In the subjects with multiple ophthalmological diseases an unknown number would have had macular degeneration secondary to another disease recorded as the principal diagnosis. Prevalence rates are consequently related to the situation where age related macular degeneration is the principal diagnosed ophthalmological disease, and are not to be taken as estimates of total prevalence of this disease. As such the prevalence rates presented in this study would be underestimates of these total prevalence rates.

This study was supported in part by a grant from the Cumberland College of Health Sciences.

1 Kahn H, Leibowitz H, Ganley J, et al. The Framingham eye study. I. Outline and major prevalence findings. $\mathrm{Am} \mathscr{f}$ Epidemiol 1977; 106: 17-32.

2 Leibowitz H, Krueger D, Maunder L, et al. The Framingham study monograph. Surv Ophthalmol 1980; suppl MayJune.

3 Sperduto R, Seigel D. Senile lens and senile macular changes in a population-based sample. Am $\mathcal{F}$ Ophthalmol 1980; 90: 86-91.

4 Podgor M, Leske M, Ederer F. Incidence estimates for len changes, macular changes, open-angle glaucoma and

5 diabetic retinopathy. Am F Epidemiol 1983; 111: 206-27. artinez G, Campbell A, Reinken J. Prevalence of ocula

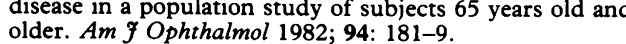
older. Am f Ophthalmol 1982; 94: 181-9.

Mitchell R, Sarks S. The impact of senile macula degeneration on the visual acuity of a large sample of hospital inpatients, 1985. Paper presented at the Seventeenth Annual Scientific Congress of the Royal Australian College of Ophthalmologists.

7 Yeates FM. Causes of binocular legal blindness in an Australian metropolitan community. Aust $f$ Ophthalmo 1983; 11: 321-3.

8 Royal Australian College of Ophthalmology. Ophthalmologists' Exchange 1987; 15(2). 